\title{
Dual responsive gelatin-based nanoparticles for enhanced 5-fluorouracil efficiency
}

\author{
M. Carmen Morán ${ }^{\mathrm{a}, \mathrm{b}}$, *, Javier Carazo ${ }^{\mathrm{a}}, \mathrm{M}$. Antònia Busquets $\mathrm{b}$, c \\ ${ }^{a}$ Departament de Bioquímica i Fisiologia-Secció de Fisiologia, Facultat de Farmàcia i Ciències de l'Alimentació- Universitat de Barcelona, Avda. Joan XXIII 27-31, 08028, Barcelona, Spain \\ ${ }^{\mathrm{b}}$ Institut de Nanociència i Nanotecnologia-IN²UB, Universitat de Barcelona, Avda. Joan XXIII 27-31, 08028, Barcelona, Spain \\ ${ }^{c}$ Departament de Farmàcia, Tecnologia Farmacèutica i Fisicoquímica-Secció de Fisicoquímica, Facultat de Farmàcia i Ciències de l'Alimentació-Universitat de Barcelona, Avda. Joan XXIII 27-31, \\ 08028, Barcelona, Spain
}

\section{A R T I C L E IN F O}

\section{Keywords:}

Nanoparticles

Gelatin

5-fluorouracil

Loading efficiency

In vitro cytotoxicity

Selectivity

\begin{abstract}
A B S T R A C T
The very slow progress in the therapeutic efficacy of the treatment of severe diseases has suggested the use of a growing need for a multidisciplinary approach to the delivery of therapeutics to targets tissues. There has been increasing effort in the design of stimuli-responsive nanomaterials that they will be developed into effective drug delivery vehicles. Most commonly, effective drug delivery is associated with nanomaterial-facilitated accumulation and/or cellular internalization. Recent studies in our lab have demonstrated that gelatin-based NPs can be considered suitable $\mathrm{pH}$ responsive devices for the effective intracellular delivery of drugs.

Concerning cancer treatment, ligands recognizing tumour-associated antigens expressed on the surface of the tumour cells have been employed. Some of the target structures suitable for tumour targeting belong to integrins which mediate cell adhesion to extracellular matrix and other cells. Interestingly, gelatin chains contain motifs such as RGD sequences that can be recognised by integrins.

In this work the inclusion of the anticancer drug 5-fluorouracil (5-FU) on these gelatin-based NPs has been projected. These NPs may provide an opportunity to increase the therapeutic effect using a dual approach by: i) targeting the therapeutic drug to the tumour cells by the action of the naturally occurring RGD-motif on gelatin and ii) minimizing the non-productive trafficking from endosomes to lysosomes by releasing the cargo using the charge reversal approach after cellular internalization. In vitro cytotoxicity experiments of NPs on tumoral and non-tumoral cell lines have reported selectivity indexes higher than 30 demonstrating a great selectivity on the mode of action as a function of the cell line and the imposed compositions.
\end{abstract}

\section{Introduction}

In recent years, there has been increasing effort in the development of stimuli-responsive nanomaterials with the hope that they will be developed into effective drug delivery vehicles. These synthetic systems utilize an assortment of endogenous or exogenous stimuli to induce a variety of responses that can facilitate targeted drug delivery. Most commonly, effective drug delivery is associated with nanomaterial-facilitated accumulation and/or cellular internalization [1]. These stimuli include small molecules, proteins, nucleic acids, peptides, electron transfer reactions, viscosity, osmotic pressure, and local environmental factors, such as $\mathrm{pH}$, temperature, or redox state [2-5].

The advances in strategies for treating a wide variety of diseases require an efficient delivery of the active compounds into the cytosol of target cells. One of the challenges for the efficient intracellular delivery of therapeutic biomolecules after their cell internalization by endocytosis is to manipulate the non-productive trafficking from endosomes to lysosomes, where degradation may occur. Recent studies in our lab have demonstrated that the gelification properties of gelatin as well as the strong dependence of gelatin ionization with $\mathrm{pH}$ makes this compound an interesting candidate to be used to the effective intracellular delivery of active biomacromolecules. Gelatin B, which an isolectric point (pI) of 4.8-5.2, is a negatively charged compound at physiological $\mathrm{pH}$. This property might be used to form nanoparticles (NPs) by interaction with oppositely charged compounds, keeping the protective structure until it enters into the cell. Once the particle enters the cell, where the $\mathrm{pH}$ of the endosome would decrease until $\mathrm{pH} 5$, gelatin $\mathrm{B}$ becomes positively charged, and due to electrostatic repulsion within positive charges the protection ceases and the entrapped drug can be re-

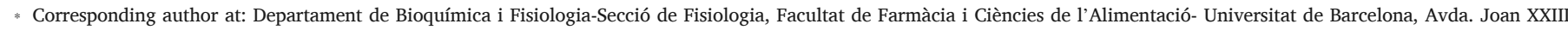
27-31, 08028, Barcelona, Spain.

Email address: mcmoranb@ub.edu (M.Carmen Morán) 
leased into the cell. This hypothesis has been recently used to the effective intracellular delivery of DNA and RNA [6,7].

In present scenario the management of cancer through drug therapies often also results in deleterious effect on the normal healthy human cells which can result in other adverse side reactions on different parts of the body. The new area of focus in treatment of cancer is by administering drug by selective toxicity. Selective toxicity in simple terms can be defined as the property of toxic substance to harm or kill a particular species of cells (i.e cancer cells) without producing any harmful effect to normal cells even if the two species are present in very close proximity [8].

For the improvement of cancer therapy, ligands recognizing tumour-associated antigens expressed on the surface of the tumour cells have been extensively employed. Some of the target structures suitable for tumour targeting belong to integrins, a family of heterodimeric cell surface receptors, consists of $\alpha$ - and $\beta$-subunits, which mediate cell adhesion to extracellular matrix and other cells [9]. These cell surface receptors are universally expressed by tumour and normal cells. However, the $\alpha \mathrm{V}$ (especially $\alpha \mathrm{V} \beta 3$ ) are highly expressed on endothelial cells lining tumour and tumour cells but poorly expressed in resting endothelial cells and most normal organs. The ligands that target these integrins show the RGD motif. Interestingly, gelatin chains contain motifs such as RGD sequences that modulate cell adhesion, thereby improving the final biological behaviour over polymers that lack this cell-recognition sites. Thus, gelatin-based NPs may provide an opportunity to increase the therapeutic effect using a dual approach by: i) targeting the therapeutic drug to the tumour tissue without the harmful effects on the normal tissue by the action of RGD-motif and ii) minimizing the non-productive trafficking from endosomes to lysosomes by releasing the cargo using the charge reversal approach after cellular internalization.

Among the therapeutic drugs, 5-fluorouracil (5-FU) still remains the only effective chemotherapeutic agent for the treatment of anal, breast, colorectal, oesophageal, stomach, pancreatic and skin cancers [10]. 5 -FU acts in several ways, but principally as a thymidylate synthase (TS) inhibitor [11]. However, primary and secondary resistance to 5-FU are common phenomena in cancer chemotherapy [12].

In the present work, the preparation and physicochemical characterization of 5-FU-based particles for the sustainable 5-FU delivery has been described. The response of the NPs co-incubated in buffers at defined $\mathrm{pHs}$ that mimic endosomal environments have been also determined. In vitro experiments have been performed in order to determine the cytotoxicity and the patterns of cell death of the NPs on selected cell lines.

\section{Materials}

Gelatin from bovine skin (gelatine type B) with gel strength $225 \mathrm{~g}$ Bloom (GB225) and $75 \mathrm{~g}$ Bloom (GB75), protamine from salmon in the sulphate salt (PS) and 5-Fluorouracil (5-FU) were purchased from Sigma and used as received.

\section{Methods}

\subsection{Preparation of proteins and 5-FU solutions}

Solutions of gelatin type B were prepared by dissolution in PBS $\mathrm{pH} 7.4$ buffer. The concentration of the gelatin solution ranged from 5 to $20 \mathrm{mg} / \mathrm{mL}$. Solutions of PS were prepared in PBS pH 7.4 buffer at concentrations 0.1 and $1.0 \mathrm{mg} / \mathrm{mL}$. 5-FU was dissolved in PBS pH 7.4 buffer (unless otherwise stated) with concentrations from 2.5 to $10 \mathrm{mg}$ / $\mathrm{mL}$. The maximum 5-FU concentration was established to $10 \mathrm{mg} / \mathrm{mL}$, due to its solubility limitations in aqueous solution [13].

\subsection{Preparation of gelatin type B(5-FU) binary mixtures}

5-FU was incorporated into the gelatin network when solutions of gelatin type B and 5-FU, previously prepared in PBS pH 7.4 (unless otherwise stated) were mixed at a ratio equal to $50 \%(\mathrm{v} / \mathrm{v})$. Then, the obtained mixtures were allowed in the refrigerator overnight, in order to promote the gel formation.

\subsection{Particle formation at the gelatin type B (5-FU)-protamine sulphate system}

Mixed gelatin (5-FU) systems $(200 \mu \mathrm{L})$ were added dropwise into the protein solution $(2 \mathrm{~mL})$ under vigorous stirring. The composition of 5-FU in the mixed systems was fixed at $50 \%(\mathrm{v} / \mathrm{v})$. Under optimal conditions, droplets from the mixed gelatin (5-FU) systems broke under magnetically stirring and instantaneously gelled into discrete particles on contact with the cationic solution. For comparative purposes, particles in the absence of 5-FU were also prepared.

\subsection{Physicochemical characterization of the gelatin type B (5-FU)- protamine sulphate nanoparticles (NPs)}

\subsubsection{Size and polydispersity}

The size was determined by dynamic light scattering (DLS) at $25^{\circ} \mathrm{C}$ with a Zetasizer Nano ZS90. The size distribution was assessed from the polydispersity index (pdI) that ranges from 0.0 for an entirely monodisperse sample to 1.0 for a polydisperse sample. The interpretation of data was performed considering the size distribution by intensity of scattered light [14].

\subsubsection{Loading efficiency}

In order to avoid some interference due to the NPs, the dispersions were centrifuged at $10.000 \mathrm{rpm}$ during $30 \mathrm{~min}$. Then, the degree of 5-FU entrapment in the NPs was determined by quantifying the free 5-FU in the supernatant solution at $267 \mathrm{~nm}$ by means of a nanophotometer.

The degree of 5-FU entrapment is expressed through the loading efficiency values. Loading efficiency (LE) is calculated by comparing the amount of 5-FU included in the NPs with the total amount during particle formation. Loading efficiency (LE) was determined with the equation:

$L E(\%)=\frac{\text { Encapsulated } 5-F U}{\text { Total } 5-F U} \times 100$

Three batches of particles were prepared in each system and the results are given as average and standard deviations.

3.5. Effect of environmental acidification on the physicochemical properties of gelatin type B (5-FU)-protamine sulphate system

Once the nanoparticle dispersions were formed at physiological $\mathrm{pH}$, two aliquots of $100 \mu \mathrm{L}$ of $0.25 \mathrm{M} \mathrm{HCl}$, were added in order to decrease the $\mathrm{pH}$ until 5.0. The obtained dispersions were gently mixed and incubated for $1 \mathrm{~h}$. The effect of environmental acidification was measured using the same parameters evaluated in Section 3.4.

\subsection{Cell cultures}

The murine Swiss albino fibroblast (3T3), the human epithelial carcinoma (HeLa) and the human breast adenocarcinoma (MCF-7) cell lines were grown in DMEM medium (4.5 g/L glucose) supplemented with $10 \%(\mathrm{v} / \mathrm{v})$ FBS, $2 \mathrm{mM}$ L-glutamine, $100 \mathrm{U} / \mathrm{mL}$ penicillin and $100 \mu \mathrm{g} /$ $\mathrm{mL}$ streptomycin at $37^{\circ} \mathrm{C}, 5 \% \mathrm{CO}_{2}$. Cells were routinely cultured in 
$75 \mathrm{~cm}$ [2] culture flasks and were trypsinised using trypsin-EDTA when the cells reached approximately $80 \%$ confluence.

\subsection{Cytotoxicity assays}

3T3 and MCF-7 cells $\left(1 \times 10^{5}\right.$ cells/ mL $)$ and HeLa $\left(5 \times 10^{4}\right.$ cells/ $\mathrm{mL})$ were seeded into the central 60 wells of a 96-well plate. After incubation for $24 \mathrm{~h}$ under $5 \% \mathrm{CO}_{2}$ at $37^{\circ} \mathrm{C}$, the spent medium was replaced in the wells with $100 \mu \mathrm{L}$ of NPs system diluted 1:1 in fresh medium supplemented with 5\% FBS.

\subsubsection{MTT assay}

In this assay, living cells reduce the yellow tetrazolium salt MTT to insoluble purple formazan crystals [15]. After cells were incubated for $24 \mathrm{~h}$ with the corresponding systems, medium was removed and $100 \mu \mathrm{L}$ of MTT in PBS ( $5 \mathrm{mg} / \mathrm{mL}$ ) diluted 1:10 in medium without FBS and phenol red was then added to the cells. The plates were incubated for a further $3 \mathrm{~h}$, after which the medium was removed. Thereafter, $100 \mu \mathrm{L}$ of DMSO was added to each well to dissolve the purple formazan product. Plates were then placed in a microtitre-plate shaker for $10 \mathrm{~min}$ at room temperature and the absorbance of the resulting solutions was measured at $550 \mathrm{~nm}$ musing a Bio-Rad 550 microplate reader. The effect of each treatment was calculated as the percentage of tetrazolium salt reduction by viable cells against the untreated cell control (cells with medium only).

\subsubsection{NRU assay}

The NRU assay determines the accumulation of the NR dye in the lysosomes of viable, undamaged cells [16]. Following exposure of cells to the NPs, medium was removed and the NPs were incubated for $3 \mathrm{~h}$ with NR dye solution $(50 \mu \mathrm{g} / \mathrm{mL})$ dissolved in medium without FBS and phenol red. Cells were then washed with sterile PBS, following by the addition of $100 \mu \mathrm{L}$ of a solution containing $50 \%$ ethanol absolute and $1 \%$ acetic acid in distilled water to extract the dye. Agitation, determination of the absorbance of the extracted solution and effect of each treatment measured at the same conditions that on 3.7.1. Section.

From the fitting of concentration-dependent viabilities curves, the corresponding half-maximal inhibitory concentration $\left(\mathrm{IC}_{50}\right)$ values for the different formulations were determined. The corresponding selectivity indexes toward cancer cells (either HeLa or MCF-7 cell line) were calculated as the following ratio:

$S 1=\frac{I C 50(\text { non }- \text { tumoral cell line })}{\text { IC50 }(\text { tumoral cell line })}$

where 3T3 fibroblasts, one of the most common non-epithelial cell line used in short- and long-term toxicological in vitro studies on cytotoxicity and biocompatibility were used as closely representative of a physiologic model cell line.

\subsection{Statistical analyses}

Experiments were performed at least three times on independent occasions unless otherwise stated. Results are expressed as means standard error of the mean (SEM). Data were analysed by PASW Statistics 18 software using one-way analysis of variance (ANOVA) with Scheffé post-hoc tests for multiple comparisons. Each experiment was performed at least three times on independent measurements. Differences were considered statistically significant at $\mathrm{p}<0.05$ or $\mathrm{p}<0.005$. In the figures significant differences were illustrated with asterisk (unless otherwise mentioned).

\section{Results and discussion}

\subsection{Preparation of gelatin type B (5-FU)-protamine sulphate NPs at physiological $\mathrm{pH}$}

The preparation of the gelatin type B (5-FU)-protamine sulphate NPs consists of the dropwise addition of highly viscous gelatin type B (5-FU) binary mixtures to protamine sulphate (PS) solutions with vigorous stirring. In this series of experiments, three variables were considered: (i) gel strength of gelatin type B (either 225 or 75 gel bloom) and gelatin concentration (ranging from 2.5 to $10 \mathrm{mg} / \mathrm{mL}$ ), (ii) PS (either 0.1 or $1.0 \mathrm{mg} / \mathrm{mL}$ ) and (iii) 5 -FU concentration (ranging from 1.25 to $5 \mathrm{mg}$ / $\mathrm{mL}$ ). For comparative purposes, in all cases, NPs in the absence of 5-FU were also prepared.

Fig. 1 summarizes the obtained results concerning the size and polydispersity of the synthesized gelatin type B (5-FU)-PS NPs. Sizes ranged from $200 \mathrm{~nm}$ to $400 \mathrm{~nm}$, which make these NPs suitable systems for the effective cellular internalization [17]. For a defined gelatin concentration, the size of the obtained NPs increases as the 5-FU concentration goes up. Concerning the influence of gelatin gel strength, type B225 might promote the formation of NPs with higher sizes than those obtained with type B75. Regarding the polydispersity values, they remained around 0.5 , independently of the compositions (Fig. 1B). Similar results were obtained for experiments carried out with PS concentration at 0.1 and $1.0 \mathrm{mg} / \mathrm{mL}$.

The degree of encapsulation of 5-FU on the proposed NPs was evaluated by means of the loading efficiency parameter (Fig. 1C). The degree of 5-FU entrapment can be modulated by varying both the gelatin type $\mathrm{B}$ and 5-FU concentrations. LE values became minimal for gelatin concentrations equal to 2.5 and $5 \mathrm{mg} / \mathrm{mL}$. For the highest gelatin concentration $(10 \mathrm{mg} / \mathrm{mL}), \mathrm{LE}$ values varied exponentially between 0 and $8 \%$ by increasing 5 -FU concentrations from 1.25 to $5 \mathrm{mg} / \mathrm{mL}$, in an almost independent way of the gelatin gel strength (either B225 or B75). Systems prepared at $1.0 \mathrm{mg} / \mathrm{mL}$ PS induced slightly lower LE values.

\subsection{Effect of $\mathrm{pH}$ on the preparation of gelatin type B (5-FU)-protamine sulphate NPs}

Studies carried out at physiological pH demonstrated limitations on the encapsulation of 5-FU, with LE values lower than $10 \%$. These values differ strongly of those obtained in the case of gelatin-based nucleic acid-containing NPs [6,7]. Using both DNA from salmon testes and three different RNA derivatives from torula yeast, NPs with LE values higher than $80 \%$ were obtained. Differences on the effectiveness on drug entrapment can be correlated with the physicochemical properties of the encapsulated drug. Thus, whereas DNA and RNA are both highly dense negative macromolecules, 5 -FU is a small molecule (MW: $130.08 \mathrm{~g} / \mathrm{mol}$ ) electrically neutral at physiological $\mathrm{pH}$. For the former derivatives, the interaction with the positively charged protamine sulphate is strongly favoured.

With the aim to promote a higher drug entrapment by increasing the negative charge density of 5-FU, the $\mathrm{pH}$ of the PBS buffer was increased until $\mathrm{pH} 8.4$ considering its pKa value, 8.02. Electrostatic interactions between oppositely charged compounds are the main driving force for the self-assembly into the proposed NPs. At either $\mathrm{pH} 7.4$ or $\mathrm{pH} 8.4$, gelatin type B (pI 4.8-5.2) acts as a negatively-charged polyelectrolyte whereas protamine sulphate (pI 12) acts as a positively-charged protein. Thus, it is expected that at this $\mathrm{pH}$ values, the ionic characteristics of both gelatin type B and PS remained unaffected. However, changes on the ionic density of 5-FU could be induced by modification of the $\mathrm{pH}$ of PBS buffer from 7.4 to 8.4 .

Fig. 2 shows representative results concerning size, polydispersity and loading efficiency values of the obtained NPs under the new condi- 

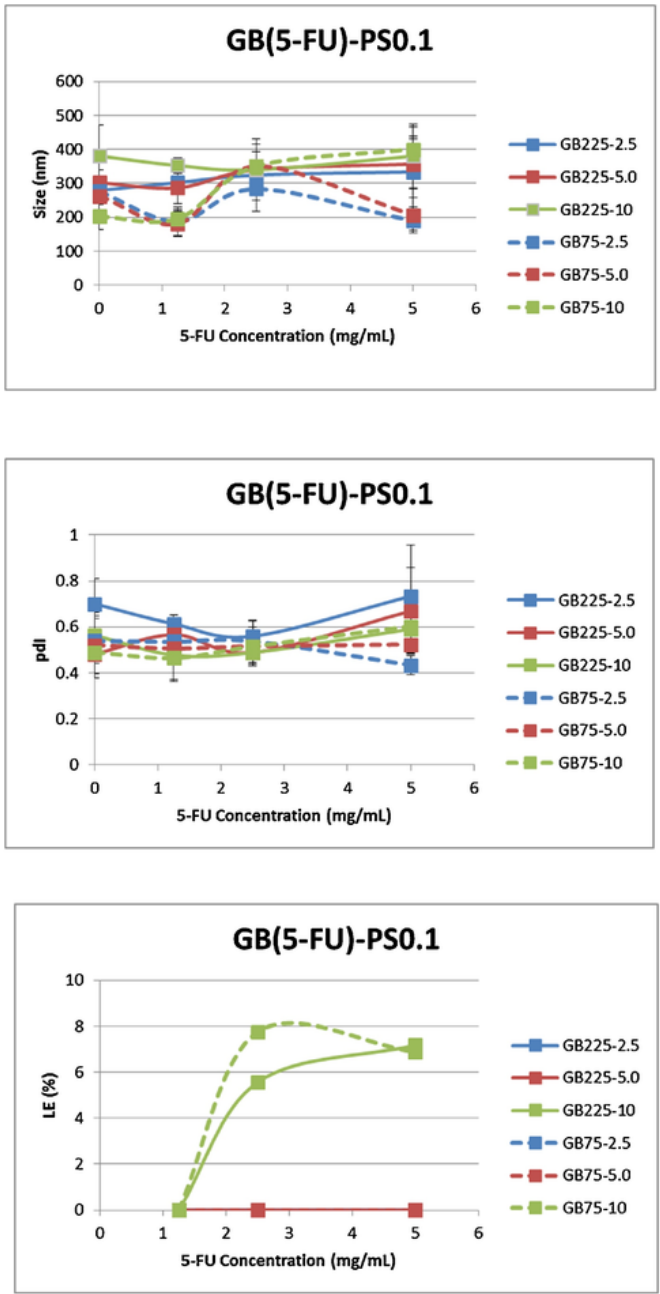

C
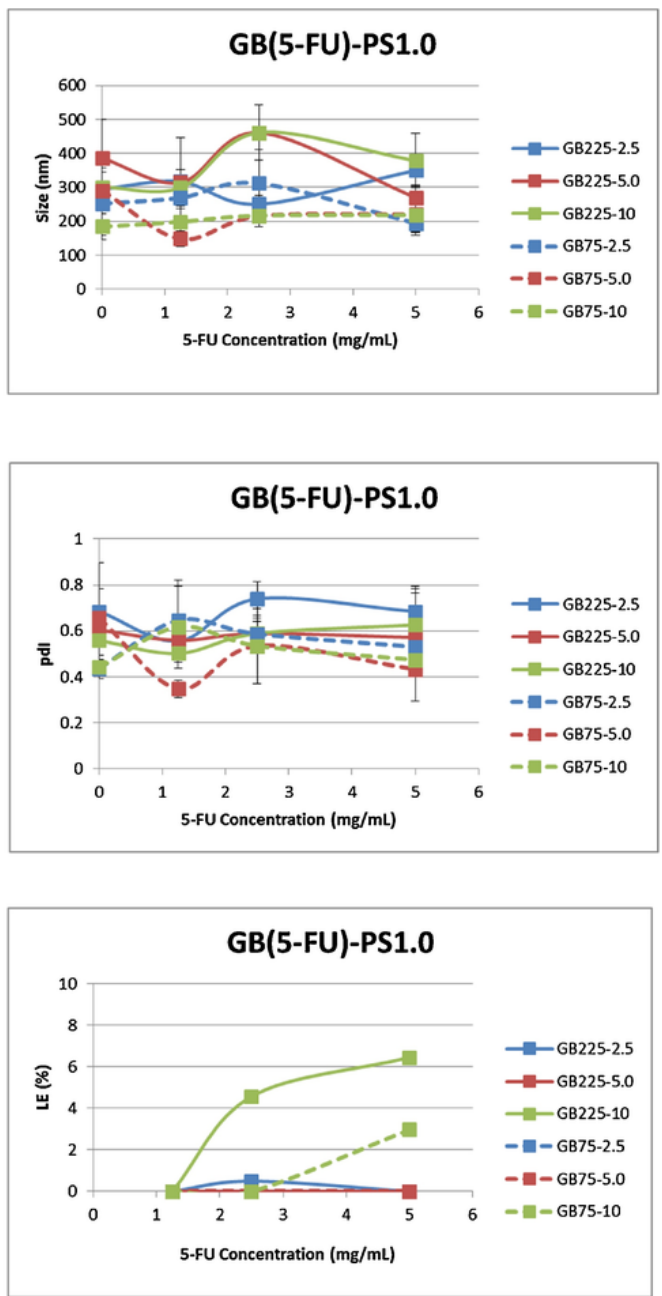

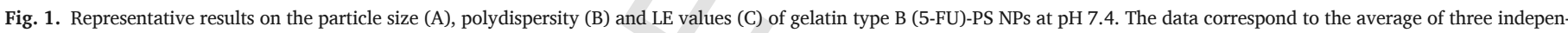
dent experiments \pm standard deviation.

tions. Not evidence changes on the size of the NPs in comparison with those obtained at $\mathrm{pH} 7.4$ were detected. In a similar way of that observed at physiological pH, NPs prepared with gelatin GB225 showed bigger dimensions than those prepared with gelatin GB75. Concerning NPs prepared with PS concentration equal to $0.1 \mathrm{mg} / \mathrm{mL}$, the minimum size values correspond to the systems containing the highest 5-FU concentration $(5.0 \mathrm{mg} / \mathrm{mL})$. For systems prepared at higher PS concentration, the NPs size result to be less sensitive to the effect of the 5-FU concentration. Fig. 2B summarizes the pdI values for the new gelatin type B (5-FU)-PS NPs prepared at $\mathrm{pH}$ 8.4. In the view of the obtained results it can be deduced that the increase in the $\mathrm{pH}$ value didn't shown a strong influence on the pdI values. In all cases, pdI values remained around 0.5-0.6.

The effect of $\mathrm{pH} 8.4$ on the loading efficiency values is shown in Fig. 2C. The degree of encapsulated 5-FU strongly depends on the gelatin concentration. Hence, for intermediate gelatin concentration $(5 \mathrm{mg} /$ $\mathrm{mL}$ ) the degree of 5-FU encapsulation exponentially decrease when the 5-FU concentration is increased, in an almost independent way of both gelatin type B and PS concentrations. However, LE exponentially increased as a function of the 5-FU concentration for either low or high gelatin concentration. The maximum loading efficiency values (40\%) correspond to systems prepared at the highest gelatin concentration (either 225 or 75$)$ at the highest 5 -FU concentration $(5 \mathrm{mg} / \mathrm{mL})$. The effect of gelatin type B concentration seems to be strongly modulated by the incorporation of 5-FU as a function of 5-FU concentration, in a fashion manner that seems to be dependent of the gelatin type B strength.

In view of the influence of both gelatin type B and 5-FU concentration on LE values (Fig. 2C), the loading efficiency values of 5-FU on the gelatin type $\mathrm{B}(5-\mathrm{FU})$ binary mixtures at both $\mathrm{pH}$ were determined. Fig. 3A demonstrates that the incorporation of 5-FU on the gelatin type $\mathrm{B}$ network for systems prepared at $\mathrm{pH} 7.4$ was almost independent of gelatin type B gel strength and concentration, and 5-FU concentration. However, when binary mixtures were prepared at $\mathrm{pH} 8.4$, the entrapment of 5 -FU is favoured, became maximum when both gelatin type $\mathrm{B}$ and 5-FU concentrations are increased (Fig. 3B).

One of the most distinguished features of gelatin is the capacity to form gels. The formation of these gels is strongly influenced by the concentration and gelatin bloom values as well as the temperature of the gel. The incorporation of 5-FU into the gelatin network was facilitated when dissolutions of gelatin type $\mathrm{B}$ and 5 -FU, previously prepared in PBS buffer, were mixed at a ratio equal to $50 \%(\mathrm{v} / \mathrm{v})$. The incubation of the binary mixtures at low temperature (approximately $10^{\circ} \mathrm{C}$ ) promoted the formation of gels for final gelatin type B concentrations ranged between 5 and $10 \mathrm{mg} / \mathrm{mL}$. For binary mixtures prepared at the lowest gelatin concentration $(2.5 \mathrm{mg} / \mathrm{mL})$, the formation of gels could not be appreciated. This observation is consistent with the 5-FU inclusion on both gelatin type B(5-FU) binary mixtures (Fig. 3) and gelatin type $\mathrm{B}(5-\mathrm{FU})-\mathrm{PS}$ NPs (Fig. $1 \mathrm{C}$ and $\mathrm{C}$ ). In the case of intermediate gelatin concentration $(5 \mathrm{mg} / \mathrm{mL})$, the decrease of $\mathrm{LE}$ values as a 

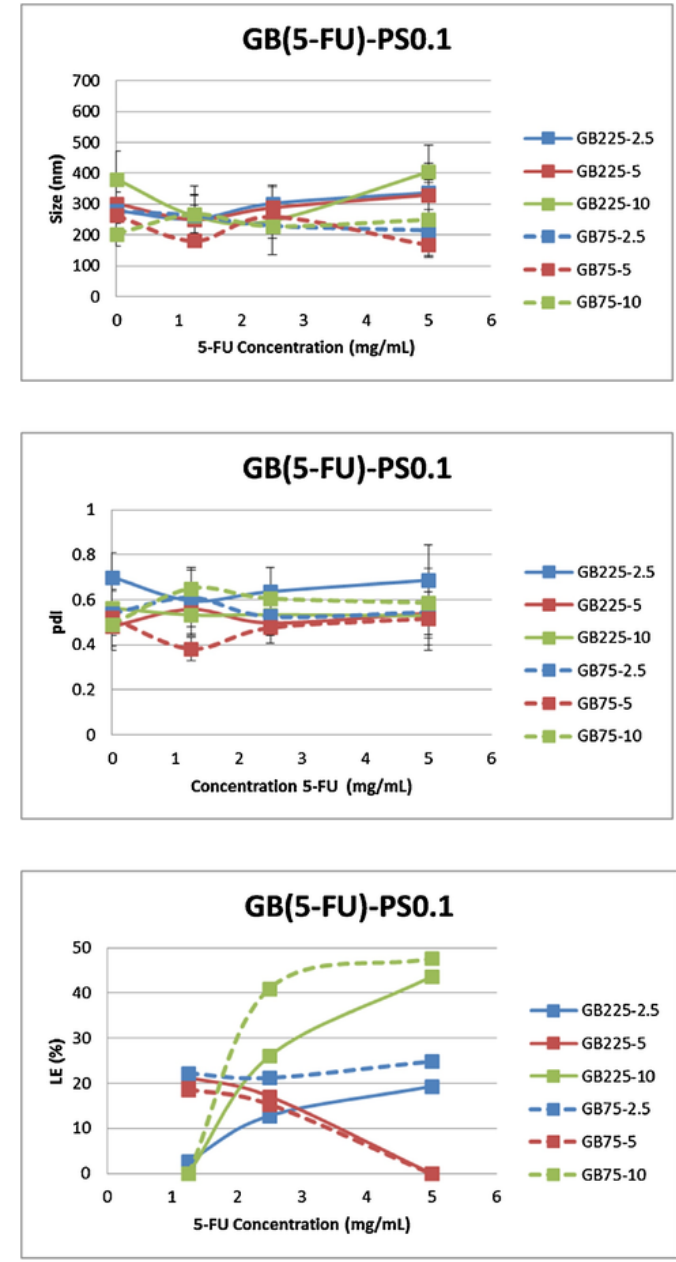
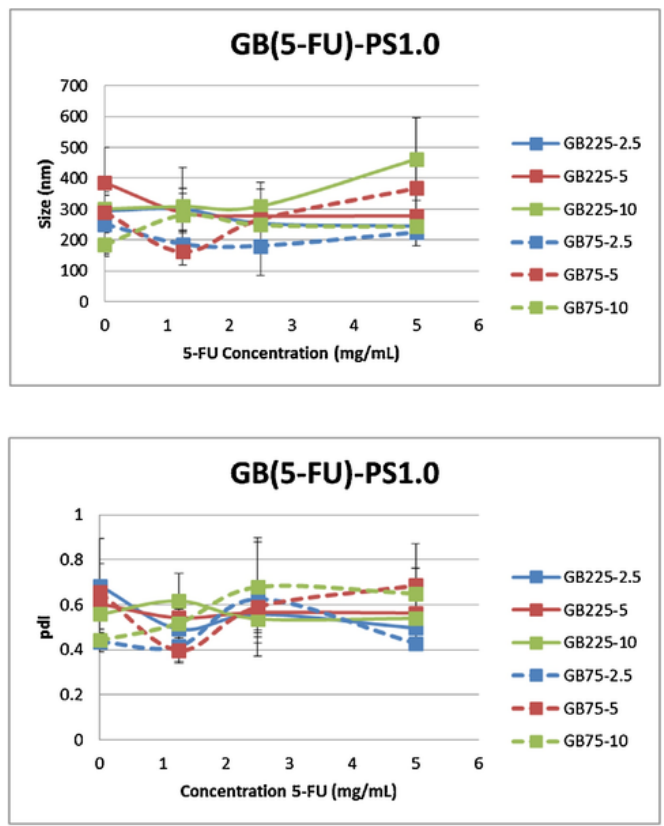

B

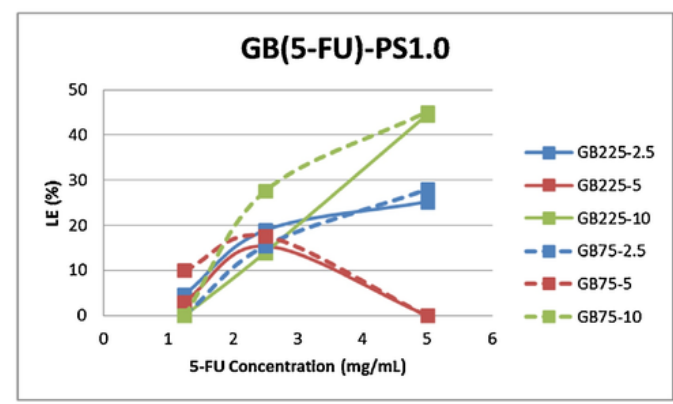

C

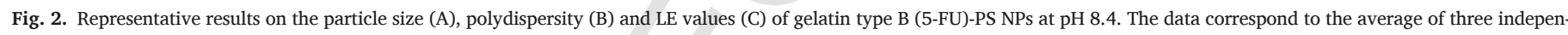
dent experiments \pm standard deviation.

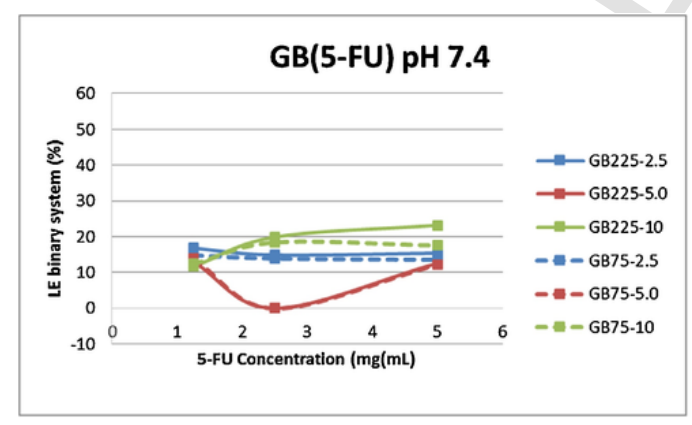

A

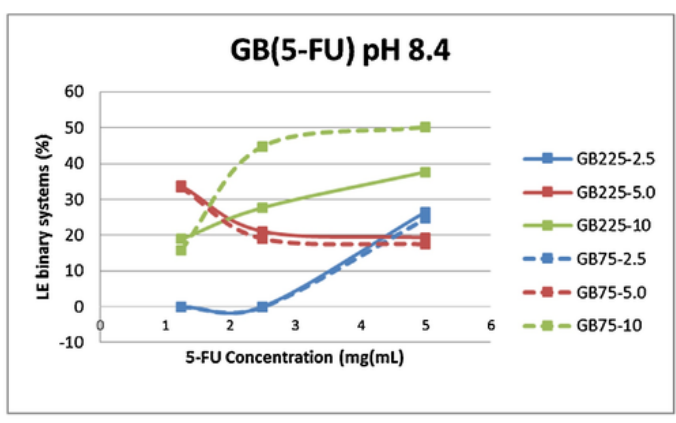

B

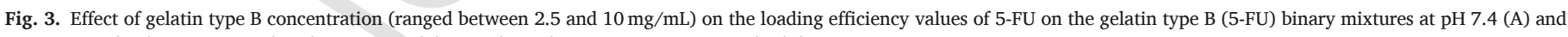
$\mathrm{pH} 8.4$ (B). The data correspond to the average of three independent experiments \pm standard deviation.

function of 5-FU concentration suggests limitations on the incorporation of 5-FU on the gelatin network. This behaviour could be correlated with some kind of electrostatically repulsion between both negative charged compounds under the assayed conditions.

In view of the obtained results, NPs systems prepared in PBS $\mathrm{pH} 8.4$ buffer at the highest gelatin type B (either 225 or 75 ) concentration were selected as optimal conditions for carried out subsequent experiments.

\subsection{Effect of the environmental acidification}

Since electrostatic interaction between oppositely charged compounds is the main driving force for the self-assembly into these NPs, it can be anticipated that a decrease in $\mathrm{pH}$, as is occurring during endosomal acidification, would influence the stability of the NPs. The decrease of the $\mathrm{pH}$ would also lead to eventually release of 5-FU from the gelatin type B (5-FU)-PS NPs. 
The effect of decrease from 8.4 to 5.0 (mimicking endosomal $\mathrm{pH}$ decrease) was evaluated as indicated in previous sections. A discrete increase on size of around $100 \mathrm{~nm}$ could be observed by acidification from extracellular to intracellular $\mathrm{pH}$ values, whereas pdI values reached levels close to 0.8 . These results were independent of all the studied conditions. LE values strongly reduced once the $\mathrm{pH}$ was lowered. In all cases, independently of gelatin type B gel strength, 5-FU and PS concentration, LE values decreased until values close to 0 (Fig. SM1 A and B).

The effect of $\mathrm{pH}$ decrease from 8.4 to 5.0 was also verified in terms of release the 5-FU (Fig. SM1C). The percentage of 5-FU released can be slightly modulated from $95 \%$ to $100 \%$ for systems prepared at 5 -FU concentration equal to 5 and $2.5 \mathrm{mg} / \mathrm{mL}$, respectively. No influence of the PS concentration (either 0.1 or $1.0 \mathrm{mg} / \mathrm{mL}$ ) on the amount of 5 -FU released was observed.

\subsection{Cytotoxicity assays}

\subsubsection{In vitro cytotoxicity of pure components in solution}

The increase of the biocompatibility and cytotoxicity of the NPs as well as their starting products constitutes a key point on this research. Previous studies in our lab have demonstrated that both gelatin type B225 and gelatin B75 are non-cytotoxic compounds. It was possible to establish the protein concentration required to inhibit cell growth by $50 \%$ compared with an untreated control $\left(\mathrm{IC}_{50}\right.$ ). This value can be defined as higher than $2000 \mu \mathrm{g} / \mathrm{mL}$ for both gelatin type B for both 3T3 and HeLa cell lines. For PS, it was found to be 670 and $1250 \mu \mathrm{g} / \mathrm{mL}$ for 3T3 (MTT and NRU, respectively) and 380 and $250 \mu \mathrm{g} / \mathrm{mL}$ for HeLa (MTT and NRU, respectively) [6].

It is well-known that 5-FU, as a chemotherapy agent, is widely chosen for the treatment of tumours. Some of its principal uses are in colorectal and pancreatic cancer, in which it has been the established form of chemotherapy for decades. It is sometimes of choice for the treatment of the aggressive inflammatory breast cancer. When the cy-
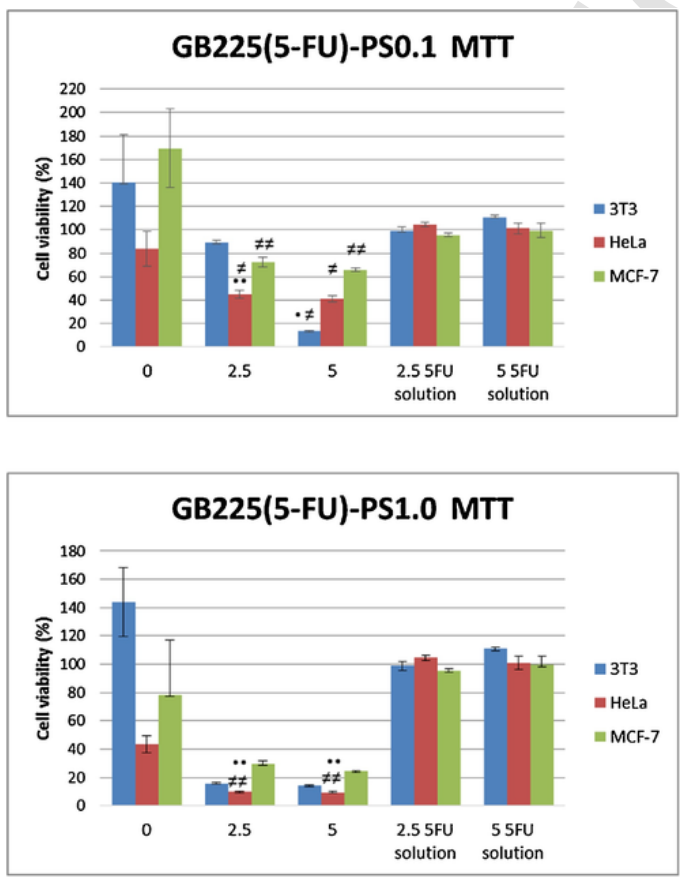

totoxicity of 5-FU has been considered, using non-tumoral 3T3 and tumoral HeLa cell lines, determined by MTT and NRU assays (Fig. SM2, panels $\mathrm{A}$ and $\mathrm{B}$ ) demonstrated that 5 -FU in solution is non-cytotoxic, even when very higher concentrations were assayed. Similar results were obtained when the cytotoxicity assays were extended to the MCF-7 cell line, a human breast adenocarcinoma (Fig. SM2 C).

These results are consistent with those described in the literature, which demonstrated that 5-FU presents the disappointing chemotherapeutic effectiveness due to side effects and multiple drug resistance [11]. Due to the high viability observed at the tested 5-FU concentration range, the $\mathrm{IC}_{50}$ has been defined as higher than $2000 \mu \mathrm{g} / \mathrm{mL}$.

\subsubsection{In vitro cytotoxicity of gelatin type B(5-FU)-protamine sulphate NPs}

Once the relative viability of the isolated components was determined, the cytotoxic effect of the gelatin type B(5-FU)-PS NPs was evaluated. For comparative purposes, particles in the absence of 5-FU, and 5 -FU in solution at the identical concentrations that those included in the NPs were also tested.

The cytotoxicity results for gelatin-based 5-FU-containing NPs as a function of the gelatin gel strength, 5-FU content and cell line are summarized in Fig. 4, when MTT method was considered. A strong dependence with the administration form on the cytotoxic responses was observed. The responses induced by 5 -FU in solution demonstrated that for this dosage form, values close to $100 \%$ were obtained by the two assayed concentrations, independently of the established cell line. Nevertheless, when the interaction of GB225(5-FU)-PS NPs with cells was considered, a strong dependence on the cell viability of the imposed conditions was obtained. For NPs prepared at PS concentration equal to $0.1 \mathrm{mg} / \mathrm{mL}$, cell viabilities ranging between 10 and $170 \%$ were obtained (Fig. 4A left). 3T3 and MCF-7 cells seem to be very sensitive to cell proliferation, with viabilities equal to 140 and $170 \%$, respectively, for NPs prepared in the absence of 5-FU. For those prepared with gelatin type B75, a similar trend was observed (Fig. 4A, right). Cell viabilities ranged between 20 and 160\%, being MCF-7 cells the most sen-
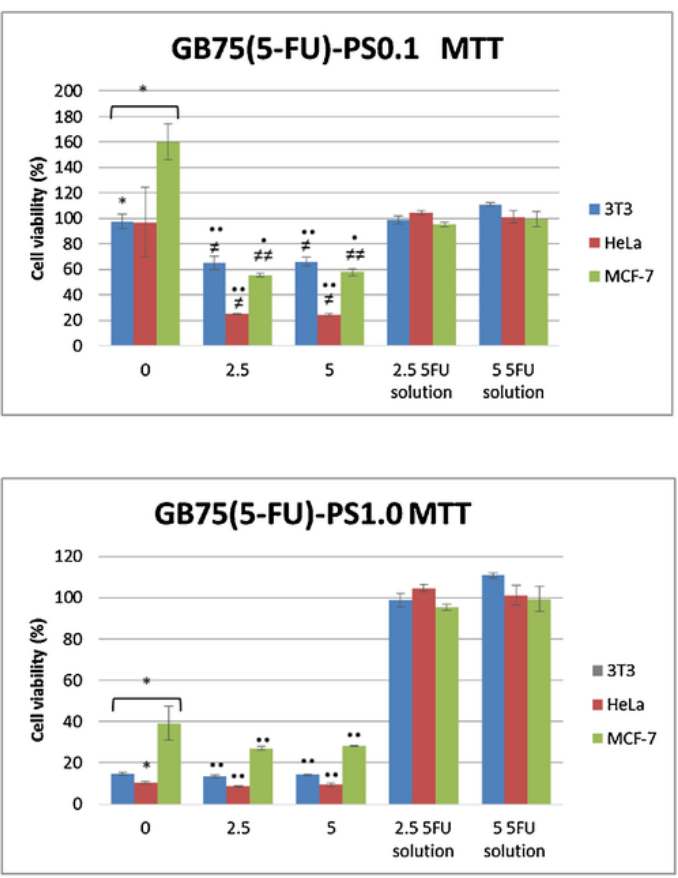

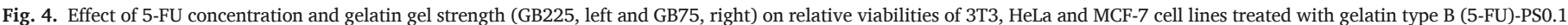

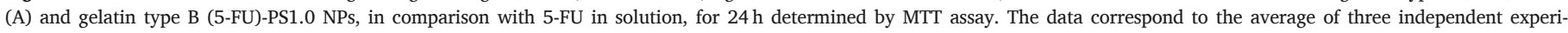

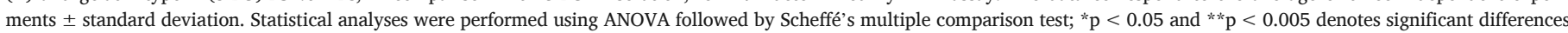

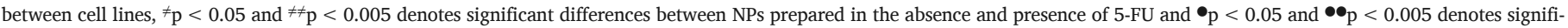
cant differences between NPs and solution, at the same 5-FU concentration. 
sitive to proliferation. Remarkably, for 5-FU-containing NPs, cell viabilities ranged between 10 and $80 \%$, in an almost independently of the gelatin gel strength. Significant differences between NPs prepared in absence and presence of 5-FU were obtained in almost all cases. Additionally, significant differences between NPS obtained in presence of 5-FU and 5-FU in solution were found.

A similar trend was observed in the case of NPs prepared at the highest PS concentration (Fig. 4B). The lowest cell viabilities values are mainly associated to the cytotoxicity of PS [6]. For NPs prepared with GB75, significant differences between NPs prepared in presence of 5-FU and 5-FU in solution were found (Fig. 4B right). In the case of gelatin GB225, these significant differences were only observed in the case of HeLa cell line (Fig. 4B left).

Taking into account that the MTT assay is a measurement of cell metabolic activity within the mitochondrial compartment [18], the obtained results suggested that 5-FU in solution involve a poor interaction with the mitochondrial compartment. However, the obtained results with gelatin-based NPs strongly suggested the uptake and internalization mediated by the nanoparticulate system.

The NRU method demonstrated poor selectivity to discriminate between NPs or 5-FU in solution (Fig. SM3). Not significant differences were found either between NPs prepared in absence or presence of 5-FU, or 5-FU incorporated into the NPs and 5-FU in solution, independently of the cell line. Cell viabilities ranged between 35 and $80 \%$ were obtained. It is interesting to note that, in all cases, the cytotoxicity responses followed the trend: 3T3 > HeLa > MCF-7. It is well established that the NRU assay measure the membrane integrity. NR dye diffuses through intact cell membranes to accumulate within lysosomes [19]. The obtained results suggested that the plasma membrane and/or lysosomal compartments of the non-tumour cell line 3T3 seems to be more sensitive to the deleterious effect of the assayed NPs or isolated compounds in comparison of those observed in the case of the two tumour cell lines. Among them, HeLa cells, however, were more susceptible than MCF-7 cell line.For a successful drug delivery system, a selective toxicity would be expected. Among the different types of cells, fibroblasts are the most abundant in various tissues and play an important role in wound healing, angiogenesis and tissue regeneration [20]. Results in this work, concerning the cytotoxicity values of 3T3 fibroblasts incubated in the presence of gelatin-based NPs (Fig. 4) suggest that these gelatin-based carriers could be considered as promising materials for the fibroblast adhesion and proliferation, as described in the literature [21]. Nevertheless, by incorporation of the corresponding antitumoral drug, the increase on the cytotoxic responses, mainly on the tumoral cell lines, would be found. Our results suggested that the required behaviour is observed in almost all cases, for which the cytotoxicity of NPs prepared in the absence of 5-FU became significantly lower than that observed when encapsulated 5-FU was considered.

From the fitting of the concentration-dependent viabilities curves, the corresponding concentration of gelatin type B (5-FU)-PS NPs, in terms of the 5-FU concentration required to inhibit cell growth by $50 \%$, compared with untreated control $\left(\mathrm{IC}_{50}\right)$ were determined. The obtained results are summarized in Table 1 . The $\mathrm{IC}_{50}$ values of the corre- sponding systems demonstrated to be a function of both cell line and endpoint method. In general, for the same cell line, MTT method demonstrated to show less cytotoxic potency than that observed in the case of NRU method. Thus, in the case of 3 T3 cell line, IC $_{50}$ values are higher than those determined by NRU method on three of the four systems. A similar trend was observed in the case of HeLa cell line. For MCF-7 cell line, however, the opposite behaviour or not differences between endpoint methods were observed. From the $\mathrm{IC}_{50}$ values can be deduced that GB225 (5-FU)-PS0.1 and GB75 (5-FU)-PS1.0 NPs provide the most and the less biocompatible systems, respectively.

The lack on cell viability where cell were incubated with NPs prepared with PS equal to $1.0 \mathrm{mg} / \mathrm{mL}$ is mainly associated to the brief cytotoxicity of PS in solution, as has been previously reported [6]. Differences between nanoparticulate systems (either GB225 or GB75) for the same PS concentration can be correlated with the capacity of the different protein systems to form weaker or stronger gelatin GB-PS complexes. In the case of the GB-PS NPs, it was expected that the higher the degree of complexation, the smaller the amount of protein that would be released in solution, an amountwhich would be able to interact with the cells and reduce their viability.

The observed differences on cell viability between nanoparticulate systems (either GB225 or GB 75) could be related with discrepancies on charge density as a function of gel strength. It has been noticed the relatively high zeta potential of gelatin type B bloom 225 over gelatin type bloom 75 which could be explained by the higher molecular weight of the former and hence higher density of carboxylic groups at the surface $[22,23]$

The superior efficacy of gelatin-based (5-FU)-containing NPs compared to free 5-FU is in line with reports in the literature. Studies demonstrated that the $\mathrm{IC}_{50}$ value of noscapine-loaded GNPs on human breast cancer cell line (MCF7) was significantly lower than that of free noscapine. ${ }^{24}$ Similarly, encapsulation of resveratrol into gelatin-based NPs enhanced its anti-cancer efficacy against NCIH460 lung cancer cells compared to free drug. ${ }^{25}$ Cytotoxicity analysis of anti-cancer drug loaded in gelatin-based NPs against different cancer cell lines showed the higher anti-cancer activity compared to free drug which might be attributed to greater endocytotic uptake of these NPs in cancer cells [24-27]. From values on Table 1 can be deduced that SI seems to be dependent on both the endpoint method and cell line type. In the case of NRU method, any selectivity was detected, independently of the cell line (either HeLa or MCF-7) and the NPs systems. In all cases, SI values are always equal or lower than 1 , demonstrating any selectivity on the mode of action. By using the MTT method, the degree of selectivity became dependent on both the cell line and the NPs systems. When MCF-7 cell line is considered, only GB225 (5-FU)-PS1.0 NPs showed slightly selectivity, in comparison of that observed in 3T3 cell line. In the case of HeLa, SI values higher than 1 where obtained for three of the four considered systems. Interestingly, in the case of GB225 (5-FU)-PS1.0 NPs, SI value higher than 30 demonstrated that this system exhibited a great selectivity for HeLa cell line, in comparison with that observed for 3T3 cell line. From the obtained results it can be deduded that the gel strenght of gelatin type B is a good factor

Table 1

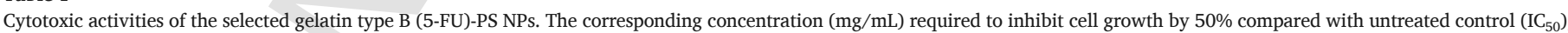

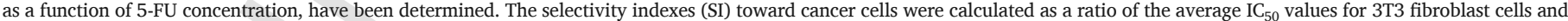
the $\mathrm{IC}_{50}$ values for the corresponding cancer cell line (either HeLa or MCF-7).

\begin{tabular}{|c|c|c|c|c|c|c|c|c|c|c|}
\hline System & $\begin{array}{l}\mathrm{IC}_{50} \\
\text { 3T3 MTT }\end{array}$ & $\begin{array}{l}\text { IC50 } \\
\text { 3T3 NRU }\end{array}$ & $\begin{array}{l}\text { IC50 } \\
\text { HeLa MTT }\end{array}$ & $\begin{array}{l}\text { IC50 } \\
\text { HeLa NRU }\end{array}$ & $\begin{array}{l}\text { IC50 } \\
\text { MCF-7 MTT }\end{array}$ & $\begin{array}{l}\text { IC50 } \\
\text { MCF-7 NRU }\end{array}$ & $\begin{array}{l}\text { SI } \\
\text { HeLa MTT }\end{array}$ & $\begin{array}{l}\text { SI } \\
\text { HeLa NRU }\end{array}$ & $\begin{array}{l}\text { SI } \\
\text { MCF-7 MTT }\end{array}$ & $\begin{array}{l}\text { SI } \\
\text { MCF-7 NRU }\end{array}$ \\
\hline GB225 (5-FU)-PS0.1 & 3.73 & 1.25 & 2.18 & 2.24 & $>5$ & $>5$ & 1.71 & 0.56 & $<0.75$ & $<0.25$ \\
\hline GB225 (5-FU)-PS1.0 & 1.84 & 0 & 0.06 & 0 & 1.43 & 4.4 & 30.16 & 1 & 1.29 & n.a. \\
\hline GB75 (5-FU)-PS0.1 & $>5$ & 0 & 1.62 & 0 & $>5$ & $>5$ & $>3.08$ & 1 & 1 & n.a. \\
\hline GB75 (5-FU)-PS1.0 & 0 & 0 & 0 & 0 & 0 & 0 & 1 & 1 & 1 & 1 \\
\hline
\end{tabular}

n.a. not applicable. 
on the mode of action of the nanoparticulate systems. As can be deduced from Fig. 4 and Table 1, NPs prepared with the lowest gel strength showed a poor selectivity between the assayed conditions than those observed with NPs prepared with the gelatin type B of highest gel strength, for which the highest SI values were obtained.

When cells are exposed to cytotoxic agents, there are various patterns of cell death that they may undergo. The two major types of cell death are apoptosis and necrosis. Necrosis is usually followed by an inflammatory response to the released cellular contents, often resulting in further tissue damage [28]. Tumours with a high incidence of necrosis rather than apoptosis show aggressive behaviour and generally have a poor prognosis [29]. Other studies have also shown a relationship between the suppression of apoptotic cell death and subsequent tumour progression in murine models [30-33]. Thus, the ability to distinguish between these two modes of cell death, especially at very early time points post-treatment, may provide useful information on the success of treatment of cancer [28].

In order to determine whether these gelatin type B(5-FU)-PS nanoparticles induced morphological alterations that could be attributed to one or another mechanism, the selected cells were incubated in the presence of the nanoparticles and then stained with the nucleic acid-binding fluorochromes AO and EB (Fig. SM4). These studies were restricted to nanoparticles prepared at PS concentration equal to $0.1 \mathrm{mg} / \mathrm{mL}$, due to the sharpest cytotoxic character of this protein at the highest assayed concentration. Under the later conditions, cells showed structurally normal orange-red nucleus as a consequence of the necrotic process.

The untreated cells mainly exhibited a green fluorescence, due to exclusion of EB but not of AO. Viable cells showed a light green nucleus with intact structure and presented punctuate orange red fluorescence in the cytoplasm, representing lysosomes stained by AO. Following treatment with gelatin-based nanoparticles, an increase in the number of apoptotic cells has been revealed. Apoptotic cells with fragmented nuclei exhibited punctate or bubbly AO distribution in cytosol. Apoptotic cells with a split nucleus and weak AO staining also exhibited punctate or aggregated AO staining in the cytosol. The most significant effects were observed with the treatments that were also the most cytotoxic in all the cell viability assays. For those cases, a slight to moderate increase in the number of cells undergoing late apoptosis was observed. These effects were 5-FU concentration-dependent, especially in the case of HeLa cells incubated with gelatin type B75 (5-FU)-PS nanoparticles (Fig. SM4).

\section{Conclusions}

The interaction between oppositely charged compounds has been the basis for developing gelatin-based NPs by mixing solutions of gelatin type B (either high or low gel strength) with solutions of protamine sulphate. The incorporation of 5-FU on the gelatin network was strongly favoured by the increment on the negative charge density in 5-FU by varying the $\mathrm{pH}$ from $7.4(\mathrm{LE}<10 \%)$ to 8.4 ( $\mathrm{LE}>40 \%)$. As a function of the imposed compositions, gelatin type B (5-FU)-PS NPs with sizes ranged from $200 \mathrm{~nm}$ to $400 \mathrm{~nm}$ and pdI (0.4-0.5) were obtained, which make these NPs suitable systems for the effective cellular internalization. NPs co-incubated in buffers at defined pHs that mimic extracellular and intracellular environments have demonstrated that 5-FU can be successfully released (95-100\%).

A fast look on the in vitro cytotoxicity studies demonstrated the strong influence of the endpoint method on the selectivity on the mode of action. The cytotoxicity results determined by the NRU method seem to be almost identical for NPs prepared in the absence and presence of 5-FU, and those observed with 5-FU in solution. Hovewer, the MTT method strongly discriminates between the action of 5-FU into the NPs and 5-FU in solution. Taking into account that the MTT assay is a mea- surement of cell metabolic activity within the mitochondrial compartment, the obtained results suggested that free 5-FU involve a poor interaction with the mitochondrial compartment. Oppositely, the uptake and internalization is strongly mediated by the nanoparticulated system. Furthermore, following treatment with gelatin-based (5-FU)-containing NPs, the increase in the number of apoptotic cells as revealed by FM studies.

For a succesful biocompatible drug delivery system, any cytotoxicity would be expected. Nevertheless, by incorporating the corresponding antitumoral drug, the increase on the cytotoxic responses, selectively target to the tumoral cell lines, would be expected. Our results demonstrated that the required behaviour is observed, for which the cytotoxicity of NPs prepared in the absence of 5-FU became significantly lower than that observed when encapsulated 5-FU was considered.

The determination of the $\mathrm{IC}_{50}$ and de corresponding SI demonstrated to be dependent onboth the endpoint method and cell line type. Thus, whereas by the NRU method, any selectivity was shown, by using the MTT method the degree of selectivity became dependent of both the cell line and the NPs systems. Considering the influence of the cell line, when MCF-7 cell line is considered, only GB225(5-FU)-PS1.0 NPs demonstrate to show slight selectivity. For HeLa, SI values higher than 1 where obtained for three of the four considered systems. Interestingly, SI values increased up to 30 for GB225 (5-FU)-PS1.0 NPs demonstrating that this system exhibited a great selectivity for HeLa cell line, in comparison with $3 \mathrm{~T} 3$ cell line.

The obtained results suggested that the proposed dual approach provides NPs with highly potent and selective non-toxic intracellular delivery properties. Current studies are focused on determining the acute intravenous toxicity of these NPs in vivo.

\section{Acknowledgments}

This research did not receive any specific grant from funding agencies in the public, commercial, or not-for-profit sectors. This work was conducted under the umbrella of COST CM1101 and MP1106 Actions.

\section{Appendix A. Supplementary data}

Supplementary material related to this article can be found, in the online version, at doi:https://doi.org/10.1016/j.colsurfb.2018.09.027.

\section{References}

[1] A.P. Blum, J.K. Kammeyr, A.M. Rush, C.E. Callmann, M.E. Hahn, N.C. Gianneschi, Stimuli-responsive nanomaterials for biomedical applications, J. Am. Chem. Soc. 137 (2015) 2140-2154.

[2] R. de la Rica, D. Aili, M.M. Stevens, Enzyme-responsive nanoparticles for drug release and diagnostics, Adv. Drug. Deliv. Rev. 64 (2012) 967-978.

[3] E. Fleige, M.A. Quadir, R. Haag, Stimuli-responsive polymeric nanocarriers for the controlled transport of active compounds: concepts and applications, Adv. Drug Deliv. Rev. 64 (2012) 866-884.

[4] S. Ganta, H. Devalapally, A. Shahiwala, M. Amiji, A review of stimuli-responsive nanocarriers for drug and gene delivery, J. Control. Release 126 (2008) 187-204

[5] N. Rapoport, Physical stimuli-responsived polymeric micelles for anti-cancer drug delivery, Prog. Polym. Sci. 32 (2007) 962-990.

[6] M.C. Morán, N. Rosell, G. Ruano, M.A. Busquets, M.P. Vinardell, Gelatin-based nanoparticles as DNA delivery systems: synthesis, physicochemical and biocompatible characterization, Colloids Surf. B 134 (2015) 156-158.

[7] M.C. Morán, I. Forniés, G. Ruano, M.A. Busquets, M.P. Vinardell, Efficient encapsulation and release of RNA molecules from gelatin-based nanoparticles, Colloids Surf. A: Physicochem. Eng. Asp. 516 (2017) 226-237.

[8] A. Albert, Selectivity Toxicity. The Physicochemical Basis of Therapy, seventh edition, Chapman and Hall, 1987.

[9] R.O. Hynes, Integrins: a family of cell surface receptors, Cell 48 (1987) 549-554.

[10] D.B. Longley, D.P. Harkin, P.G. Johnston, 5-fluorouracil: mechanisms of action and clinical strategies, Nat. Rev. Cancer 3 (2003) 330-338.

[11] W. Huanwen, L. Zhiyong, S. Xiaohua, R. Xinyu, W. Kai, L. Tonghua, Intrinsic chemoresistance to gemcitabine is associated with constitutive and laminin-induced phosphorylation of FAK in pancreatic cancer cell lines, Mol. Cancer 8 (2009) 125 . 
[12] R.M. Madera, M. Müllera, G.G. Stegera, Resistance to 5-Fluorouracil, Gen. Pharmacol. 31 (1998) 661-666.

[13] R.L. Sastre, M.D. Blanco, C. Teijón, R. Olmo, J.M. Teijón, Preparation and characterization of 5-Fluorouracil-loaded poly( $\varepsilon$-Caprolactone) microspheres for drug administration, Drug Dev. Res. 63 (2004) 41-53.

[14] http://www.materials-talks.com/blog/2014/10/23/ polydispersity-what-does-it-mean-for-dls-and-chromatography/.

[15] T. Mosmann, Rapid colorimetric assay for cellular growth and survival: application to proliferation and cytotoxicity assays, J. Immunol. Methods 65 (1983) 55-65.

[16] E. Borenfreund, J.A. Puerner, Toxicity determined in vitro by morphological alterations and neutral red absorption, Toxicol. Lett. 24 (1985) 119-124.

[17] J. Rejman, V. Oberle, I.S. Zuhorn, D. Hoekstra, Size-dependent internalization of particles via the pathways of clathrinand caveolae-mediated endocytosis, Biochem. J. 377 (2004) 159-169.

[18] M.V. Berridge, P.M. Herst, A.S. Tan, Tetrazolium dyes as tools in cell biology: new insight into their cellular reduction, Biotechnol. Annu. Rev. 11 (2005) 127-152.

[19] G. Repetto, A. Peso, J.L. Zurita, Neutral red uptake assay for the estimation of cell viability/cytotoxicity, Nat. Protoc. 3 (2008) 1125-1131.

[20] B. Sarker, R. Singh, R. Silva, J.A. Roether, J. Kaschta, R. Detsch, D.W. Schubert, I. Cicha, A.R. Boccaccini, Evaluation of fibroblasts adhesion and proliferation on alginate-gelatin crosslinked hydrogel, PLoS One 9 (2014), e107952.

[21] K.W. Wissemann, B.S. Jacobson, Pure gelatin microcarriers: synthesis and use in cell attachment and growth of fibroblast and endothelial cells, In Vitro Cell. Dev. Biol 21 (1985) 391-401.

[22] A.O. Elzoghby, Gelatin-based nanoparticles as drug and gene delivery systems: reviewing three decades of research, J. Control. Release 172 (2013) 1075-1091.

[23] M. Nahar, D. Mishra, V. Dubey, N.K. Jain, Development, characterization, and toxicity evaluation of amphotericin B-loaded gelatin nanoparticles, Nanomed.: NBM 4 (2008) 252-261.
[24] J. Madan, N. Dhiman, S. Sardana, R. Aneja, R. Chandra, A. Katyal, Long-circulating poly(ethylene glycol)-grafted gelatin nanoparticles customized for intracellular delivery of noscapine: preparation, in-vitro characterization, structure elucidation, pharmacokinetics, and cytotoxicity analyses, Anticancer Drugs 22 (2011) 543-555.

[25] S. Karthikeyan, N.R. Prasad, A. Ganamani, E. Balamurugan, Anticancer activity of resveratrol-loaded gelatin nanoparticles on NCI-H460 non-small cell lung cancer cells, Biomed. Prev. Nutr. 3 (2013) 64-73.

[26] R.B. Badisa, S.F. Darling-Rees, P. Joseph, J.S. Cooperwood, L.M. Latinwo, C.B. Goodman, Selective cytotoxic activities of two novel synthetic drugs on human breast carcinoma MCF-7 cells, Anticancer Res. 29 (2009) 2993-2996.

[27] R.B. Badisa, D.A. Mina, L.M. Latinwo, K.F.A. Soliman, Selective anticancer activity of neurotoxin 1-methyl-4-phenylpyridinium on non-small cell lung adenocarcinoma A549 cells, Anticancer Res. 34 (2014) 5447-5452.

[28] T. Bezabeh, M.R.A. Mowat, L. Jarolim, A.H. Greenberg, I.C.P. Smith, Detection of drug-induced apoptosis and necrosis in human cervical carcinoma cells using $1 \mathrm{H}$ NMR spectroscopy, Cell. Death Differ. 8 (2001) 219-224.

[29] J.V. Moore, Death of cells and necrosis of tumors, in: CS Potten (Ed.), Perspectives on Mammalian Cell Death, Oxford University Press, Oxford, 1987, pp. 295-324.

[30] P. Naik, J. Karrim, D. Hanahan, The rise and fall of apoptosis during multistage tumorigenesis: down-modulation contributes to tumor progression from angiogenic progenitors, Genes Dev. 10 (1996) 2105-2116.

[31] S. Cory, D.L. Vaux, A. Strasser, A. Harris, J.M. Wand Adams, Insights from bcl-2 and myc: malignancy involves abrogation of apoptosis as well as sustained proliferation, Cancer Res. 59 (1999), 1685S \pm 1692S.

[32] C. Renvoize, A. Biola, M. Pallardy, J. Breard, Apoptosis: identification of dying cells, Cell. Biol. Toxicol. 14 (1998) 111-120.

[33] A.J. McGahon, S.J. Martin, R.P. Bissonnette, A. Mahboubi, Y. Shi, R.J. Mogil, W.K. Nishioka, D.R. Green, The end of the (cell) line: methods for the study of apoptosis in vitro, Methods Cell. Biol. 46 (1995) 153-185. 